\title{
Comportamiento del Profesor, Intensidad y Tiempo Efectivo de las Clases de Educación Física en una escuela pública: Un acercamiento ala realidad \\ Teacher's Behavior, Intensity and Effective Time of Physical Education Classes in a Public School: An Approach to Reality \\ *Cristian Retamal-Valderrama, *Pedro Delgado-Floody, *Miguel Espinoza-Silva, **Daniel Jerez-Mayorga \\ *Universidad de La Frontera (Chile), **Universidad Andres Bello (Chile)
}

Resumen. Actualmente se recomienda que los estudiantes participen en Actividad Física Moderada a Vigorosa (AFMV) al menos el 50\% del tiempo empleado en la clase de Educación Física (EF). Por tal motivo el objetivo fue identificar el nivel de Actividad Física (AF), comportamiento del Profesor y tiempo efectivo de las clases de EF, en una escuela pública de Chile. Durante dos meses, se observaron 14 clases de EF y a tres profesores (2 hombres y 1 mujer), los alumnos, presentaban edades entre 6 a 10 años. La observación se realizó considerando solo las clases que tuvieran una duración de 90 minutos y que fueran realizadas en un día sin climas extremos como lluvia, calor intenso o contaminación ambiental. Se evaluó 1) el porcentaje de tiempo de actividad física del estudiante 2) los contenidos de la clase 3) la participación del profesor. Mediante el sistema de observación del tiempo de instrucción de condición física. Como principales resultados encontramos que el tiempo dedicado a una AFMV fue de un 32\% de la clase de EF. Además, el 68\% fue destinado a la práctica libre y el 90.6\% el profesor no promovió el esfuerzo. Como conclusión, los resultados de la investigación indican que la AFMV fue más baja que la recomendada y el mayor tiempo de la clase de EF no se promueve el esfuerzo dentro de los escolares.

Palabras clave: actividad física, educación física, escolares, profesor, intensidad.

Abstract. It is recommended that students are engaged at least 50\% of their Physical Education (PE) classes in moderate to vigorous physical activity (MVPA). Thus, the objective was to identify the physical activity (PA) level, professor's behavior and effective time of PE classes in a public school in Chile. Through two months, $14 \mathrm{PE}$ classes and three teachers were observed. Observed students were between 6 and 10 years old. Only the classes that lasted 90 minutes and were not run in extreme conditions like rain, intense heat or environmental contamination were analyzed. We evaluated 1) student's PA in percentage of time 2) class contents 3) teacher's participation. Everything was assessed through the system of observation of the time of instruction of the physical condition. The time dedicated to MVPA was 32\% of the class. $68 \%$ was destined to the free practice. $90.6 \%$ the teacher did not promote the effort. The results indicate that the PA was significantly lower than the recommendation and in majority of time the PE teacher did not promote the students' effort.

Keywords: physical activity, physical education, children, teacher, intensity.

\section{Introducción}

Mundialmente, la falta de actividad física (AF) se ha convertido en uno de los factores de riesgo más importante en el desarrollo de enfermedades crónicas no transmisibles (Blanchard, Shilton, \& Bull, 2013), sus bajos niveles ha incrementado la prevalencia de obesidad infantil (Ebbeling, Pawlak, \& Ludwig, 2002), y ha afectado diversas variables psicosociales como la depresión (De Wit et al., 2010), baja autoestima y alteración de la imagen corporal (Delgado et al., 2017)

Por otra parte, la práctica deAF tiene efectos potencialmente beneficiosos para la salud mental, tanto para la depresión, reducción de la ansiedad, aumento de la autoestima, el funcionamiento cognitivo y el rendimiento académico (González \& Portolés, 2015; Marques, Gómez, Martins, Catunda, \& Sarmento, 2016). Lamentablemente, la realidad demuestra consistentemente que los estudiantes que participan en los programas típicos de educación física (EF) de primaria realizan bajos niveles AF de moderada a vigorosa (AFMV) (Bevans, Fitzpatrick, Sanchez, Riley, \& Forrest, 2010; Coe, Pivarnik, Womack, Reeves, \& Malina, 2006; Scruggs et al., 2003). Esto debido a diferentes factores como el contexto de la escuela, las características técnicas del profesor, el tamaño de la clase, los equipos, instalaciones, duración de la clase y el número de clases por semana (Honório, Martins, Torres, Cardoso, \& Costa, 2016; Smith, Lounsbery, \& McKenzie, 2014; Sutherland et al., 2016). Además, uno de los obstáculos identificados para disminución de la práctica de AFMV durante la clase es la cantidad de tiempo dedicado a las tareas de gestión. Esto incluye el tiempo dedicado a las tareas administrativas (por ejemplo, la asistencia, la creación de equipos) o manejo de la conducta. Estudios multicéntricos han evaluado los programas típicos de EF de primaria y se han encontrado que entre el $21.1 \%$ y el 23.1\% del tiempo de clase se dedica a las tareas de gestión (Bevans et al., 2010).

Actualmente, los niños pasan gran parte de su tiempo en la escuela,

Fecha recepción: 13-03-18. Fecha de aceptación: 10-07-18 Pedro Delgado Floody

pedrodelgadofloody@gmail.com lo cual representa un entorno propicio para el aumento del gasto energético por AF y la promoción de un estilo de vida saludable y activo (Fernández, González, Toja , \& Carreiro, 2016). Sin embargo, la presión que se ejerce sobre los establecimientos educacionales para mejorar el rendimiento académico, ha reducido el tiempo disponible para la práctica de AF, incrementando así el tiempo sedentario de los escolares (Aguilar, Martino, \& Espinoza, 2015; Onofre, 2016). Incluso el poco tiempo de EF destinado en los establecimientos educacionales, no está siendo aprovechado en su totalidad, siendo este ocupado en actividades principalmente de baja intensidad. Así lo demuestran dos estudios en Latinoamérica, los cuales reflejan la realidad de las clases de EF, donde solo se acumuló un promedio de 38.2\% y 29.2\% de AFMV (JenningsAburto et al., 2009; Pérez, 2016).

Por lo anteriormente señalado el objetivo de la investigación fue identificar el nivel de AF, comportamiento del Profesor y tiempo efectivo de movimiento de las clases de EF, en una escuela pública de Temuco. Utilizando el sistema de observación de tiempo de instrucción de condición física (SOFIT), un instrumento diseñado para evaluar los niveles de AF durante las clases.

\section{Materiales y métodos}

El presente estudio sigue un diseño metodológico descriptivo de corte transversal, con una muestra de conveniencia no probabilística utilizando SOFIT para observar 14 clases de EF mixtas de primero a cuarto año básico, con 480 alumnos (230 mujeres y 250 hombres), completando 270 observaciones por cada clase de 90 minutos (3.780 intervalos de observación) de una Escuela Pública de Temuco, Chile. Con un promedio de 30 alumnos por clase, con proporciones similares de niños y niñas. 3 profesores de educación física, 2 Hombres y 1 Mujer.

Se consideraron las clases observadas con una duración de 90 minutos, durante días sin restricción ambiental por contaminación y sin lluvia, ni temperaturas extremas. Los participantes, tanto padres, alumnos y profesores firmaron un consentimiento informado, el cual autoriza la observación de las clases de EF. 


\section{Procedimiento}

Para evaluar el nivel de AF del estudiante, contenido de la clase y comportamiento del profesor, se utilizó el instrumento SOFIT, el cual es un instrumento de observación para evaluar la calidad de la clase de EF y los niveles de actividad del estudiante (Mckenzie, Sallis, \& Nader, 1992). Este instrumento se ha validado con otros métodos objetivos, como monitores de frecuencia cardiaca y acelerómetros. Para este estudio, los observadores fueron entrenados siguiendo el estándar del protocolo SOFIT, memorizando las definiciones operativas de los códigos y aprendiendo la técnica, procedimientos y medidas de fiabilidad.

Este instrumento está compuesto de intervalos de observación de 20 segundos, guiado por un audio Mp3.

Los primeros diez segundos se observan tres variables.

Actividad del estudiante: 1 = acostado; 2 = sentado; 3 = parado; 4 = caminando; $5=$ muy activo. (AFMV surge de la combinación de los códigos 4 y 5)

Contenido de la clase: Contenido general (G), Contenido de conocimiento $(\mathrm{C})$, condición física $(\mathrm{F})$, práctica de habilidades $(\mathrm{H})$, juegos (J), y participación libre (L)

Participación del profesor: Promueve que la actividad física sea ejecutada durante la clase (I), Promueve que la actividad física sea ejecutada por fuera de la clase $(\mathrm{O})$, No promueve actividad física $(\mathrm{N})$.

En los siguientes diez segundos se registran los códigos para cada variable. Produciéndose 3 observaciones y tres registros cada minuto, para un total de 270 observaciones en una clase de 90 minutos.

Al inicio de la observación, se deben seleccionar a 5 niños por clase, 2 hombres y 2 mujeres, el $5^{\circ}$ solo se observa si cualquiera de los 4 primeros sale del campo visual del observador. La selección de los estudiantes es al azar a medida que llegan a la clase, se seleccionan los estudiantes 4, 8, 12, 16 y 20 para clases con menos de 25 estudiantes, y 5, 10, 15, 20 y 25 si el número es mayor.

Cada estudiante es observado por un periodo de 4 minutos continuos, siguiendo los intervalos del protocolo, en los primeros $16 \mathrm{minu}-$ tos de la clase se produce una ronda completa de observación de los 4 estudiantes, repitiendo el procedimiento hasta terminada la clase.

\section{Análisis estadístico}

Para el análisis de las variables SOFIT (intensidad, contenido de la clase y comportamiento del profesor), se calculó el porcentaje de tiempo destinado para cada uno de los ítems, mediante el software Excel para Windows10, presentándolo como figuras graficas en porcentaje de tiempo.

\section{Resultados}

En la Figura 1, se puede observar que del $100 \%$ de las clases de EF Observadas (90 minutos por clase), en promedio solo 32\% del tiempo fue destinado a una AFMV, lo cual se obtiene sumando las variables Caminando y Muy activo, aquello indica que solo $4.4 \%$ del tiempo está destinado a una intensidad muy activa, mientras que la mayor cantidad de tiempo los niños se encuentran sentados 47.9\% y Caminando 27.6\% (Figura 1).

En la Figura 2 se puede observar que del $100 \%$ de las clases de EF Observadas (90 minutos por clase) el 68\% del tiempo está destinado a la práctica libre, mientras que el resto está destinado a los demás contenidos como el General (9.2\%), Conocimiento (11.2\%), Condición Física (1.7\%), Habilidades (3.3\%) y Juegos (6.0\%) (Figura 2).

En la Figura 3 se puede observar que del $100 \%$ de las clases de EF Observadas (90 minutos por clase), el $90.6 \%$ de la clase, el profesor no promueve el esfuerzo o la práctica de actividad física, mientras que el resto de la clase (9.4\%) si promueve el esfuerzo. Por otra parte se observó que el profesor nunca destinó tiempo a promover la práctica de actividad física fuera de la clase o del establecimiento (Figura 3).

\section{Discusión}

El objetivo de la investigación fueidentificar el nivel deAF, compor-

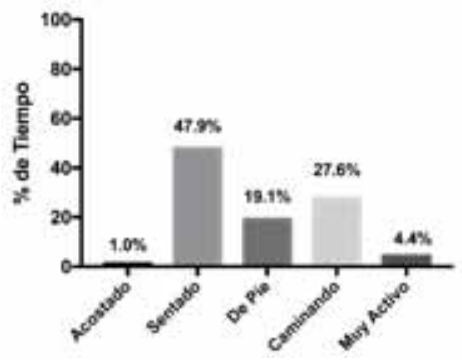

Figura 1. Tiempo de Actividad Física de los Estudiantes, Según Intensidad. Fuente: elaboración propia basada en los datos obtenidos en el estudio.

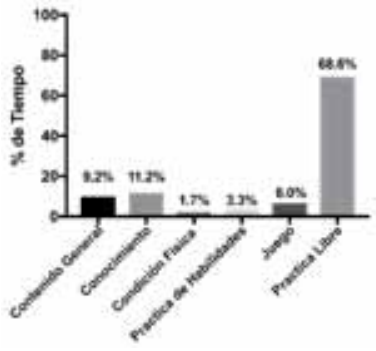

Figura 2. Tiempo de las Clases Según su Contenido. Fuente: elaboración propia basada en los datos obtenidos en el estudio.

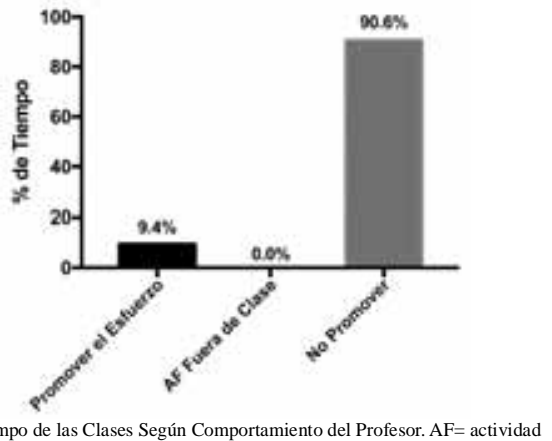
física. Fuente: elaboración propia basada en los datos obtenidos en el estudio.

tamiento del Profesor y tiempo efectivo de AF de las clases de EF, en una escuela pública de Temuco, Chile. Utilizando SOFIT, un instrumento diseñado para evaluar los niveles de AF durante las clases. La escuela representa un entorno propicio para el aumento del gasto energético por AF (Silva et al., 2018; Tremblay et al., 2016) y la promoción de un estilo de vida saludable y activo (Fernández et al., 2016; Lonsdale et al., 2013). Lamentablemente los hallazgos encontrados en este estudio muestran una preocupante realidad, principalmente al tiempo dedicado a una AFMV durante las clases de educación física, la cual alcanzó niveles muy bajos, llegando a un promedio de $32 \%$. Resultados concordantes con un estudio que recopiló datos de 38 países, donde más del $60 \%$ de los niños no cumplen con el tiempo recomendado de AFMV (Tremblay et al., 2016).

Estos resultados, se pueden explicar por diferentes motivos, principalmente por el contenido de la clase y el comportamiento del profesor (Gharib et al., 2015), los cuales fueron predominantemente dedicados a la práctica libre de los alumnos (68.6\%) y a la no promoción del esfuerzo (90.6\%), lo cual sugiere que el comportamiento del profesor, específicamente en el clima motivacional y las estrategias utilizadas, conduce a una baja motivación del alumnado hacia la práctica de AF (Cabello, Moyano , \& Tabernero, 2018). Por otra parte, el contenido de las clases no presentaba muchos elementos lúdicos como el juego (6.0\%), práctica de habilidades (3.3\%) o la condición física (1.7\%), disminuyendo así los beneficios que estas actividades generan en el auto concepto físico, la autoestima y el desarrollo integral del niño (Gil-Espinosa, Romance, \& Nielsen, 2018). Asimismo, la literatura señala que existen muchos factores que determinan la intensidad de las actividades durante el horario escolar, incluidas las características técnicas de los maestros, el 
tamaño de la clase, instalaciones, duración de la clase programada y el número de clases por semana (Hernández-Álvarez, Del-Campo-Vecino, Martínez-de-Haro, \& Moya-Morales, 2010; Navas \& Soriano, 2016).

En Chile, existen evidencias similares, como los reportados por un estudio realizado en 549 escolares chilenos de establecimientos municipales de la Región Metropolitana, donde se monitoreó por podómetros durante la clase de EF, tanto clases realizadas por especialistas, como por profesores generalistas. Los resultados más importantes de este estudio mostraron que durante la clase de EF de 90 minutos, el tiempo en el cual los niños practicaron AFMV es sumamente bajo (14.4\%). El hecho de que en las clases de EF la proporción de tiempo en AFMV sea tan baja, ha sido analizado en otros estudios a nivel mundial. Por ejemplo dos estudios realizados en Latinoamérica, reportaron un promedio de AFMV en clases de EF de 29.2\% y un 38.7\%, cada uno (JenningsAburto et al., 2009; Pérez, 2016). Por otra parte, en México se midió el nivel de AF durante 63 clases de EF y recreos de una escuela pública, mediante el instrumento SOFIT. Reportándose que durante los periodos de recreos, los niños realizaban mayor AFMV, siendo esta un 18\% más que la de una clase de EF tradicional (Hall-Lopez, Ochoa-Martinez, Burruel, Ortiz, \& Bunuel, 2017). Otros estudios desarrollados en Europa, donde se monitoreó a través de acelerometría a 59 niños preescolares durante 4 días de clases, indicó que en promedio, el 82.31\% del tiempo empleado en la escuela se asignó a tareas sedentarias. Aproximadamente 7.53\% del tiempo fue destinado a una AFMV (Vale, Santos, Silva, Soares-Miranda, \& Mota, 2011). En España también usando acelerómetros, se reportó que los alumnos en una clases de EF de 120 minutos, realizaron AFMV el 9.3 \% de la clase (Martínez, Contreras, Aznar, \& Lera, 2012), un valor incluso inferior al de chiley Latinoamérica.

En el ámbito de la participación del profesor durante la clase, se reportó que solo 9.4\% del tiempo de la clase de EF, se promovió el esfuerzo, mientras que el resto del tiempo el profesor no lo hacía(90.6\%). Los resultados de este estudio sugieren que la motivación en la clase de EF por parte del profesor, es fundamental para su desarrollo, tanto por la intensidad que ésta requiere, como por el clima motivacional el cual conlleva a una mayor y mejor AF. Así lo demuestra un estudio realizado en España, donde 294 estudiantes fueron analizados, mediante la Escala de Locus (Goudas, Biddle, \& Fox, 1994), la cual mide la motivación hacia la clase de EF y las pruebas deALPHA-Fitness de Alta Prioridad para determinar la condición física de los estudiantes (Ruiz et al., 2011). Los resultados reportaron que existe una asociación positiva entre la motivación hacia la EF y la capacidad cardiorrespiratoria, por lo que los autores recomiendan que los profesores de EF deban dar una mayor importancia a la motivación. Otro estudio que analizó la relación entre los diferentes niveles de feed-back con la motivación intrínseca del alumnado en la clase de EF, reportó que la motivación de los alumnos en clases de EF se ve afectada positivamente por el papel del feed-back del docente, concretamente del feed-back de carácter positivo (MorenoMurcia, Huéscar, Peco, Alarcón, \& Cervelló, 2013).

Por otra parte, un estudios realizado en Chile y Alemania (Carcamo, Wydra, Hernandez, \& Martinez, 2017) indica que la actitud, el grado de importancia y conformidad que tienen los alumnos hacia la clase de EF mayoritariamente es positivo, incluso muestra que los chilenos tienden a darle un mayor grado de importancia a la asignatura, por lo que esta característica positiva de los alumnos debe ser aprovechada de mejor forma para mejorar los aprendizajes de los niños y el nivel de AF.

También se pudo observar que los niños durante la clase, destinaban mucho tiempo de espera para poder participar de algún juego, circuito o actividad, esto debido a la cantidad de alumnos participando de una sola actividad. En relación a esto se recomienda dividir el curso en diferentes actividades simultáneas, o generar mayor cantidad de hileras, para optimizar así el tiempo y privilegiar el movimiento. Esto debido a que la escuela es el principal lugar y quizás el único donde los niños puedan practican algún tipo de AF (Fernández et al., 2016).

Se recomienda utilizar el instrumento SOFIT como una medida objetiva, económica y fácil de aplicar, para evaluar la efectividad de las clases de EF, generando estrategias remediales y reflexión en torno a la importancia de optimizar el poco tiempo asignado a laAF, espacialmente en estos tiempos donde la inactividad física y la obesidad afectan a un gran número de la población nacional y mundial.

\section{Limitaciones}

Las principales limitaciones fueron no incluir mayor número de establecimientos educacionales en el estudio, lo que habría permitido realizar comparaciones y tener datos que reflejaran aún más la realidad. Tampoco se utilizó otro sistema de medición más directo, como por ejemplo acelerómetro que permitiera comparar los valores obtenidos.

\section{Conclusiones}

Los resultados encontrados muestran una preocupante realidad en la clase de EF, donde predominaron los contenidos de limitada AF, dejando de lado las actividades lúdicas mediante el juego, condición física y la práctica de habilidades. Además, con una participación casi ausente del profesor durante la clase, el cual no promovía el esfuerzo, ni mucho menos la práctica de AF fuera de ella. Los resultados, reportaron que el $68 \%$ del tiempo de la clase, fue destinado a la práctica libre, lo cual indica que los alumnos se auto regulan por si mismos en la intensidad y tiempos de actividad. Por otra parte, se muestra una baja prevalencia del juego, lo cual provoca que la clase no sea un lugar propicio para las actividades lúdicas, dirigidas y planificadas, privilegiando el contenido de conocimiento y relegando la condición física y la práctica de habilidades a un segundo plano. Finalmente podemos concluir que existe una urgente necesidad de mejorar los tiempos de la clase deEF, mediante una mayor participación del profesor, transformándose en un agente promotor y motivador de la práctica de AF y hábitos saludables, disminuyendo así sustancialmente las actividades administrativas y el contenido de conocimiento dando con ello mayor énfasis a la condición física, la práctica de habilidades y el juego.

\section{Conflicto de intereses}

No existe conflicto de interés.

\section{Financiación}

Esta investigación contó con el apoyo de la Unidad de Gestión Pedagógica de la Municipalidad de Temuco.

\section{Agradecimientos}

El equipo de investigación agradece al Plan de desarrollo de la motricidad como potenciador de los aprendizajes y estado nutricional, de la Unidad de Gestión Pedagógica de la Municipalidad de Temuco.

\section{Referencias}

Aguilar, N., Martino, P., \& Espinoza, M. (2015). Objectively measured physical activity and sedentary behaviour patterns in Chilean preschool children. Nutr Hosp, 32, 2606-2612.

Bevans, K. B., Fitzpatrick, L. A., Sanchez, B. M., Riley, A. W., \& Forrest, C. (2010). Physical education resources, class management, and student physical activity levels: a structure-process-outcome approach to evaluating physical education effectiveness. $J$ Sch Health, 80(12), 573-580. doi: 10.1111/j.1746-1561.2010.00544.x

Blanchard, C., Shilton, T., \& Bull, F. (2013). Global Advocacy for Physical Activity (GAPA): global leadership towards a raised profile. Global Health Promotion, 20(4_suppl), 113-121. doi: 10.1177/1757975913500681

Cabello, A., Moyano , M., \& Tabernero, C. (2018). Procesos psicosociales en Educación Física: actitudes, estrategias y clima motivacional percibido (Psychosocial processes in Physical Education: attitudes, strategies, and perceived motivational climate). 
Retos, 34, 19-24.

Carcamo, J., Wydra, G, Hernandez, C., \& Martinez, C. (2017). Actitudes hacia la educación física: Grados de importancia y conformidad según escolares de Chile y Alemania. Una mirada intercultural (Attitudes towards physical education: Degrees of importance and conformance among schoolchildren in Chile and Germany. Retos, 32, 158-162.

Coe, D. P., Pivarnik, J., Womack, C., Reeves, M., \& Malina, R. (2006). Effect of Physical Education and Activity Levels on Academic Achievement in Children. Med Sci Sports Exerc, 38(8), 1515-1519. doi: 10.1249/01.mss.0000227537.13175.1b

De Wit, L., Luppino, F., van Straten, A., Penninx, B., Zitman, F., \& Cuijpers, P. (2010). Depression and obesity: A meta-analysis of community-based studies. Psychiatry Res, 178(2), 230-235. doi: 10.1016/j.psychres.2009.04.015

Delgado, P., Martínez , C., Caamaño, F., Jerez, D., Osorio, A., García, F., \& Latorre, P. (2017). Insatisfacción con la imagen corporal y su relación con el estado nutricional, riesgo cardiometabólico y capacidad cardiorrespiratoria en niños pertenecientes a centros educativos públicos. Nutr Hosp, 34(5), 1044-1049.

Ebbeling, C. B., Pawlak, D. B., \& Ludwig, D. S. (2002). Childhood obesity: public-health crisis, common sense cure. The Lancet, 360(9331), 473-482. doi: 10.1016/s0140-6736(02)09678-2

Fernández, M., González, M., Toja , B., \& Carreiro, F. (2016). Valoración de la escuela y la Educación Física y su relación con la práctica de actividad física de los escolares. Retos, 31(312-315).

Gharib, H., Galavíz, K. I., Lee, R. E., Safdie, M., Tolentino, L., Barquera, S., \& Lévesque, L. (2015). The Influence of Physical Education Lesson Context and Teacher Behaviour on Student Physical Activity in Mexico. Retos, 28, 160-164.

Gil-Espinosa, F., Romance, Á., \& Nielsen, A. (2018). Juego y actividad física como indicadores de calidad en Educación Infantil Retos, 34, 252-257.

González, J., \& Portolés, A. (2015). Recomendaciones de actividad física y su relación con el rendimiento académico en adolescentes de la Región de Murcia. Retos, 24, 100-104.

Goudas, M., Biddle, S., \& Fox, K. (1994). Perceived locus of causality, goal orientations, and perceived competence in school physical education classes. Br JEduc Psychol, 64(3), 453-463. doi: 10.1111/ j.2044-8279.1994.tb01116.x

Hall-Lopez, J. A., Ochoa-Martinez, P. Y., Burruel, R. Z., Ortiz, L. R. M., \& Bunuel, P. S. L. (2017). Moderate-to-vigorous physical activity during recess and physical education among mexican elementary school students. Retos, 31, 137-139.

Hernández-Álvarez, J., Del-Campo-Vecino, J., Martínez-de-Haro, V., \& Moya-Morales, J. (2010). Perception of Exertion in Physical Education and its Relationship to Guidelines on Physical Activity. Rev.int.med.cienc.act.fís.deporte.

Honório, S., Martins, J., Torres, B., Cardoso, J., \& Costa, A. M. (2016). Influence of sociological aspects on the level of physical activity in physical education students. 2016, 10(3), 12. doi: 10.14198/ jhse.2015.103.07

Jennings-Aburto, N., Nava, F., Bonvecchio, A., Safdie, M., GonzálezCasanova, I., Gust, T., \& Rivera, J. (2009). Physical activity during the school day in public primary schools in Mexico City. Salud Publica Mex, 51, 141-147.

Lonsdale, C., Rosenkranz, R. R., Peralta, L. R., Bennie, A., Fahey, P., \& Lubans, D. R. (2013). A systematic review and meta-analysis of interventions designed to increase moderate-to-vigorous physical activity in school physical education lessons. Preventive Medicine, 56(2), 152-161. doi: https://doi.org/10.1016/j.ypmed.2012.12.004

Marques, A., Gómez, F., Martins, J., Catunda, R., \& Sarmento, H. (2016). Association between physical education, school-based physical activity, and academic performance: a systematic review (Asociación entre la educación física, la actividad física en la escuela, y el rendimiento académico: una revisión sistemática). Retos; Núm. 31 (2017).
Martínez, J., Contreras, O. R., Aznar, S., \& Lera, Á. (2012). Children’s physical activity levels measured by accelerometer: all day physical activity vs physical education classes. Rev Psicol Deporte, 21(1), 117-123.

Mckenzie, L., Sallis, J., \& Nader, P.(1992). SOFIT: System for observing fitness instruction time. Journal of teaching in physical Education, 11(2), 195-205.

Moreno-Murcia, J. A., Huéscar, E., Peco, N., Alarcón, E., \& Cervelló, E. (2013). Relations of feed-back and teacher communication barriers with in-trinsic motivation among adolescent students in physical education. Anal. Psicol., 29, 257-263.

Navas, L., \& Soriano, J. (2016). Analysis of the reasons to practice or not extracurriculum physical activities and its relationship with physical self-concept in Chilean students. Revista Iberoamericana de Psicología del Ejercicio y el Deporte.

Onofre, M. (2016). A Qualidade da Educação Física como Essência da Promoção de uma Cidadania Ativa e Saudável(Calidad de la Educación Física como esencia para promover la ciudadanía activa y saludable). Retos, 31, 328-333.

Pérez,A. (2016). Impacto de la clase de educación física sobre la actividad moderada y vigorosa en niños de primaria. Revista Mexicana de Investigación en Cultura Física y Deporte, 1(1), 148-171.

Ruiz, J. R., Espana Romero, V., Castro Pinero, J., Artero, E. G., Ortega, F. B., Cuenca, M., . . Castillo, M. J. (2011). Bateria ALPHAFitness: test de campo para la evaluacion de la condicion fisica relacionada con la salud en ninos y adolescentes. Nutr Hosp, 26(6), 1210-1214.

Scruggs, P.W., Beveridge, S. K., Eisenman, P. A., Watson, D. L., Shultz, B. B., \& Ransdell, L. B. (2003). Quantifying physical activity via pedometry in elementary physical education. Med Sci Sports Exerc, 35(6), 1065-1071. doi: 10.1249/01.MSS.0000069748.02525.B2

Silva, D. A. S., Chaput, J.-P., Katzmarzyk, P. T., Fogelholm, M., Hu, G., Maher, C., . . . Tremblay, M. S. (2018). Physical Education Classes, Physical Activity, and Sedentary Behavior in Children. Medicine and science in sports and exercise, 50(5), 995-1004. doi: 10.1249/mss.0000000000001524

Smith, N. J., Lounsbery, M. A. F., \& McKenzie, T. L. (2014). Physical Activity in High School Physical Education: Impact of Lesson Context and Class Gender Composition. Journal of Physical Activity and Health, 11(1), 127-135. doi: 10.1123/jpah.2011-0334

Sutherland, R., Campbell, E., Lubans, D. R., Morgan, P. J., Okely, A. D., Nathan, N., . . . Wiggers, J. (2016). Physical education in secondary schools located in low-income communities: Physical activity levels, lesson context and teacher interaction. J Sci Med Sport, 19(2), 135-141. doi: 10.1016/j.jsams.2014.12.003

Tremblay, M. S., Gonzalez, S. A., Katzmarzyk, P. T., Onywera, V. O., Reilly, J. J., \& Tomkinson, G. (2016). Introduction to the Global Matrix 2.0: Report Card Grades on the Physical Activity of Children and Youth Comparing 38 Countries. Journal of Physical Activity and Health, 13(11 Suppl 2), S85-S86. doi: 10.1123/jpah.20160641

Vale, S., Santos, R., Silva, P., Soares-Miranda, L., \& Mota, J. (2011). Relationship of objective measurement of physical activity during school hours and BMI in preschool children. Int J Pediatr Obes, 2 , 37-38.

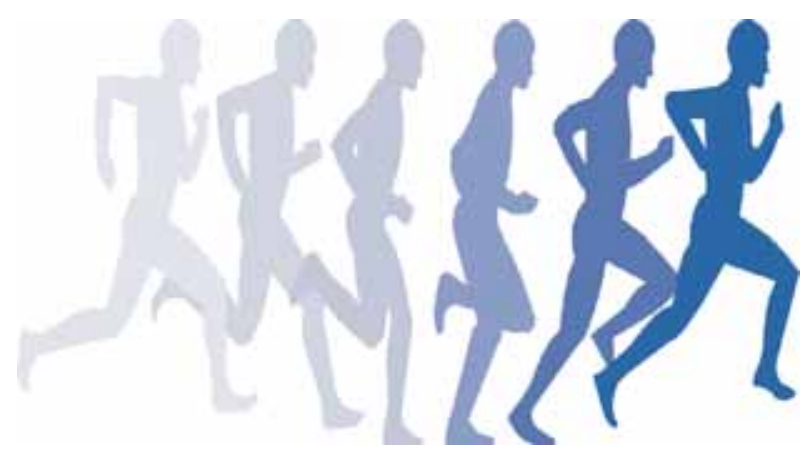

